\title{
The Effect of an Online Self-Help Cognitive Behavioural Intervention for Insomnia on Negative Affect and Paranoia: A Randomised Controlled Trial
}

\author{
Alexander J. Scott*, Georgina Rowse ${ }^{\dagger}$ and Thomas L. Webb ${ }^{\ddagger}$
}

Background: Sleep and mental health go hand-in-hand, with problems sleeping being associated with a variety of mental health difficulties. Recently, insomnia has been linked with the experience of paranoia, a relationship that is likely to be mediated by negative affect. Given these links, the present research aimed to test whether a self-help intervention designed to improve sleep can also improve negative affect and paranoia.

Method: Participants were recruited from a mailing list of University staff and were randomly allocated to one of three conditions; a wait-list control group, an active control group who completed a sleep diary each day for 6 weeks, and an experimental group who received an online self-help intervention targeting sleep problems alongside the same sleep diary. Levels of insomnia, negative affect, and paranoia were measured at baseline, immediately post-intervention, and 4- and 18-weeks post-intervention.

Results: There were no significant differences between the groups on levels of insomnia, negative affect, and/or paranoid thinking at post-intervention, 4-weeks, or the 18-week follow-up. However, a relatively large number of participants dropped out of the study, particularly in the intervention group, which meant that the primary analysis was underpowered.

Conclusion: Due to a high level of participant dropout, the findings from the present research are inconclusive, and suggest that retaining participants in trials of online interventions is a significant challenge that needs to be addressed in future research.

Keywords: Self-help; Insomnia; Negative affect; Paranoia; CBTi; Randomised Controlled Trial

Paranoid thoughts are both distressing and relatively common in the general population. For example, (Freeman et al., 2005) investigated the experience of paranoia in a sample of 1,202 members of the general population. Approximately one third of the sample experienced paranoid thoughts, many of which caused distress, ranging from milder expressions (e.g., people at work are talking about me) to more serious paranoid thoughts often seen in clinical groups (e.g., my food is being poisoned by the police). Similarly, insomnia (i.e., problems getting to sleep and/or staying asleep) is also relatively common, with around one third of people experiencing at least one symptom of insomnia (Ohayon, 2002). Furthermore, there may be an association between

\footnotetext{
* School of Health and Related Research (ScHARR), The University of Sheffield, Sheffield, UK

+ Clinical Psychology Unit, The University of Sheffield, UK * Department of Psychology, The University of Sheffield, UK Corresponding author: Alexander J. Scott (alex.scott@sheffield.ac.uk)
}

paranoia and sleep, as many, if not all mental health difficulties are associated with problems sleeping (Baglioni et al., 2016; Benca et al., 1992), and in recent years a number of empirical studies have reported that the experience of insomnia is closely related to paranoid thinking. For example, insomnia is associated with paranoia in both clinical and non-clinical populations (Freeman et al., 2009), an effect that is likely mediated by affective experiences (Freeman et al., 2009, 2010, 2012; Scott et al., 2017) and emotion regulation (Grezellschak et al., 2017; Rehman et al., 2018). This link between insomnia, negative affect, and the experience of paranoia raises an important question; can interventions primarily aimed at improving insomnia also reduce paranoia?

\section{Current Attempts to Improve Paranoia by Reducing Insomnia}

There are a range of treatment options for people experiencing insomnia (for reviews, see Riemann \& Perlis, 2009; Smith et al., 2002; Unbehaun et al., 2010). However, perhaps the most effective and widely used interventions are those based on the principles of Cognitive Behavioural 
Therapy for insomnia (CBTi, Okajima et al., 2011; Trauer et al., 2015) which can be offered both face-to-face (Trauer et al., 2015) or remotely via the internet (Cheng \& Dizon, 2012; Zachariae et al., 2016). Myers, Startup, and Freeman (2011) investigated the efficacy of four sessions of CBTi aimed at reducing levels of insomnia in those experiencing persistent persecutory delusions. The authors reported a large effect of the intervention on insomnia, as well as paranoia, depression, and anxiety immediately post-treatment as well as 1-month later. Although reporting promising early effects, this study does have several methodological limitations; notably, the absence of a control group and the use of unblinded assessments. In an effort to address some of these limitations, Freeman et al. (Freeman et al., 2015) randomised 50 participants with psychosis spectrum diagnoses to receive either standard care alone (consisting of antipsychotic medication and contact with the local clinical team), or CBT for insomnia (in addition to standard care) in an assessor blind randomised controlled trial. Although this trial was of superior methodological quality to its forerunner (Myers et al., 2011), and the authors again reported large effects of the intervention on insomnia, this time there was little subsequent improvement on measures of paranoia and other symptoms of psychosis, with effect sizes generally in the small range.

\section{Self-Help for Insomnia as a Promising Route}

Face-to-face CBT has many benefits including contact with a therapist and control over the therapeutic process; however, there are limitations. For example, face-to-face CBT is time consuming for the therapists and services providing it (Aschim et al., 2011; Wiebe \& Greiver, 2005), is often offered at great cost and, thus, has limited availability (Cavanagh, 2014; Shapiro et al., 2003). Given that most service providers do not have the luxury of abundant time and finances; self-help can provide a viable alternative to face-to-face interventions. Self-help interventions have been shown to be effective in treating insomnia (Ho et al., 2015; van Straten \& Cuijpers, 2009), psychosis spectrum experiences (Scott et al., 2015), and common mental health problems such as depression and anxiety (Cuijpers et al., 2010; Gellatly et al., 2007). Given; (i) the links between insomnia and negative affect (Freeman et al., 2009, 2010, 2012); (ii) between negative affect and paranoia (Fowler et al., 2012; Lincoln et al., 2009, 2010); and (iii) the beneficial effects of self-help interventions in dealing with insomnia and mental health problems (Cuijpers et al., 2010; Gellatly et al., 2007; Ho et al., 2015; Scott et al., 2015), it seems possible that a self-help intervention primarily designed to improve sleep could; (i) reduce negative affect; and/or (ii) offer a cost-effective and accessible way to reduce paranoid thinking.

\section{The Present Research}

The present research involved a randomised controlled trial investigating the impact of a self-help intervention for insomnia on the experience of negative affect and paranoia in a non-clinical sample. The aim was to test the hypothesis that interventions designed to improve sleep subsequently improve both negative affect and paranoia. The effects of the intervention were compared against two control conditions; an active control group (who were simply asked to complete a sleep diary each day) and a wait-list control group (who received no intervention). It was predicted that participants allocated to the intervention condition would report reduced levels of insomnia, negative affect, and paranoia when compared to participants in the active and wait-list control conditions.

\section{Method \\ Trial Design}

This was a single-centre, assessor-blind parallel group randomised controlled trial comprising of three groups; (i) an intervention group who received an online CBTi intervention; (ii) an active control group who completed an online sleep diary for the duration of the intervention; and (iii) a wait-list control group who received no intervention during the study. The trial was retrospectively registered on the International Standard Randomised Controlled Trial Number registry (ISRCTN18384206).

\section{Participants and Procedures}

Eligibility criteria

Participants were eligible for inclusion if they were at least 18 years of age. Exclusion criteria for the trial included; (i) problems sleeping due to a physical complaint (e.g., back pain); (ii) currently taking medication for a mental health problem or sleep complaint; or (iii) currently receiving psychological therapy for a mental health problem or sleep complaint.

\section{Participants}

Participants were recruited from a mailing list comprising employees at The University of Sheffield who had not opted out of a distribution list set up to advertise opportunities to take part in research studies. Recruitment began in April 2014, and the final participant completed follow-up measures in August 2015. All participants were initially recruited via an email invitation, from here they were directed to the study website where they registered their interest for the study and completed the online baseline measures.

\section{Procedures}

After participants registered their interest in the study and completed the baseline measures, they were randomised to one of three groups; (i) an intervention group who received an online CBTi intervention for experiences of insomnia; (ii) an active control group who completed an online sleep diary for the duration of the intervention; and (iii) a wait-list control group who received no intervention during the study. Participants in the intervention group were given on screen instructions on how to navigate the website as well as an overview of the intervention and what was expected of them. Participants assigned to the sleep diary group were given information on how to complete the online sleep diary each day. Participants in the wait-list 
control were told that they would be offered the intervention after they had completed the final follow-up measure. The intervention lasted 6 weeks, after which participants were asked to complete post-intervention measures; they were then followed up 4- and 18-weeks later.

\section{Outcome Measures}

The Insomnia Subscale of the Sleep-50

Insomnia was measured using the insomnia subscale of the Sleep-50 questionnaire (Spoormaker et al., 2005). Participants were asked to indicate (on a 4-point scale) the extent to which 8 statements about their sleep applied to them within the last four weeks, with higher scores indicating more severe insomnia. Four items were concerned with initially falling to sleep (e.g., "I worry so much it prevents me from falling asleep" and "I find it difficult to fall asleep"), and four items were concerned with staying asleep (e.g., "I wake up during the night" and "I sleep lightly"). The insomnia subscale of the Sleep-50 is a valid and reliable tool to measure insomnia, and has been shown to have high internal consistency and good testretest reliability (Spoormaker et al., 2005). In the present research, the insomnia subscale (alpha $=0.72$ ) proved internally reliable.

The Short-Form of the Depression, Anxiety, Stress Scale (DASS-21)

Negative affect was measured using the shortened form of the Depression, Anxiety, and Stress Scale (DASS-21, Henry $\&$ Crawford, 2005). Participants were asked to indicate (on a 4-point scale), the extent to which they agreed with statements assessing facets of negative affect over the past week, with higher scores indicating higher levels of negative affect. For example, 7 statements were concerned with feelings of depression (e.g., "I felt down-hearted and blue" and "I couldn't seem to experience any positive feeling at all"). A further 7 statements asked participants about feelings of anxiety (e.g. "I was aware of the action of my heart" and "I experienced breathing difficulty". Finally, 7 questions aimed to explore feelings of stress (e.g. "I found it difficult to relax" and "I was intolerant of anything that kept me from getting on"). In the present research, total negative affect was calculated using the sum total of items in the DASS-21. The DASS-21 has been validated for use in both clinical and non-clinical populations and has been found to be a reliable and valid measure of negative affect (Henry $\&$ Crawford, 2005). In the present research, alpha $=0.92$.

The Green Paranoid Thoughts Scale - Part B (GPTS-B)

Paranoid thoughts were measured using Part B of the Green Paranoid Thoughts Scale (GPTS-B, Green et al., 2008) that focuses on persecutory thoughts. Participants were asked to indicate the extent to which they agreed with sixteen statements over the previous month (e.g., "Certain individuals have had it in for me" and "It was difficult to stop thinking about people wanting to make me feel bad") on a 5-point scale where higher scores indicated higher levels of paranoia. A total score was computed by summing each item of the GPTS-B. The GPTS has been validated for use in both clinical and non-clinical populations and has been shown to have good internal consistency and validity (Green et al., 2008). In the present research, alpha $=0.96$.

\section{Intervention and Comparators}

The Sleep Healthy Intervention

An online self-help intervention designed to designed to target factors that contribute to the formation and maintenance of insomnia symptoms was adapted from that developed by Lancee, van den Bout, van Straten, and Spoormaker (2012), which was in turn developed from a preceding RCT of a self-help CBTi intervention (van Straten et al., 2009) and informed by established treatment manuals for insomnia (Morin, 1993). The intervention consisted of six weekly modules based on the principles of CBTi, including; psychoeducation materials, sleep hygiene, stimulus control, relaxation exercises, sleep restriction, cognitive restructuring, and paradoxical intention. Each module was designed to be used in conjunction with subsequent modules, so that participants could draw upon a wide range of methods and techniques by the end of the intervention programme. Table $\mathbf{1}$ describes each weekly module of the intervention, alongside practical examples from the intervention, and a rationale explaining the insomnia factor(s) that each module targeted.

\section{Week One}

In the first week (and then throughout the six-week intervention), participants were asked to use a sleep diary to record and monitor their sleep each day, and received psychoeducation about sleep (e.g., the stages of sleep, functions of sleep), as well as the prevalence of problems sleeping and some possible reasons for this. Crucially, participants were introduced to the idea of 'sleep hygiene', a term used to describe the different practices, environments, and habits that are conducive, or not, to good sleep (Perlis \& Youngstedt, 2000). In week one participants were given educational materials detailing both good, and bad sleep hygiene practices (e.g., substance use including alcohol and caffeine, use of mobile devices before bed)). Poor sleep hygiene does not necessarily cause people to experience insomnia; however, it can contribute to its maintenance (Sharma \& Andrade, 2012), and is therefore an integral component of many interventions targeting insomnia (Morin, 1993).

\section{Week Two}

In week two, participants were encouraged to use stimulus control techniques, a technique pioneered by Bootzin (1972), to re-associate the bed and bedroom with sleep. For example, in week two, participants were given information on a range of habits and behaviours that are not conducive to sleeping. Then, participants asked to formulate plans using a planner built into the study website to encourage the adoption of good sleep habits (e.g., "I will get into bed at 10pm each night this week"), and to discourage behaviours that weaken the association between the bed(room) and sleep (e.g., "I will ensure I do not watch television in bed this week"). Week two also introduced 
Table 1: An Overview of the 'Sleep Healthy' Intervention.

\begin{tabular}{lll} 
Week & $\begin{array}{l}\text { Module ele- } \\
\text { ments }\end{array}$ & Module description \\
\hline One & Psychoeducation & $\begin{array}{l}\text { Participants were provided with information on sleep, sleep } \\
\text { problems, and sleep hygiene. }\end{array}$
\end{tabular}

Sleep diary

Two control/sleep hygiene

Progressive muscle relaxation

Sleep diary

Three

Sleep restriction

Imaginative relaxation

Sleep restriction review

Sleep diary

Five

Challenging misconceptions

Sleep restriction review

Sleep diary

Six

Participants were asked to complete a diary each day throughout the intervention. Participants were asked to record the time that they went to bed, estimated sleep onset time, total time spent asleep, number nightly awakenings, rise times, and how refreshed they felt in the morning.

The aim of this module was to re-associate the bed(room) with sleeping by encouraging the adoption of good sleep habits, and discouraging habits that exacerbate problems with sleep onset/maintenance.

Participants were instructed to contract and relax muscle groups progressively over time in a set order.

Continued from previous week.

Participants were instructed to go to bed later than usual to ensure that the time spent in bed is spent sleeping (rather than tossing and turning, watching television etc.). Sleep restriction was used in conjunction with stimulus control (see above), and was titrated so that sleep is more or less restricted (in $30 \mathrm{~min}$ ute blocks), depending on the time taken to fall asleep.

Participants were encouraged to visualize a peaceful and relaxing scene (of their own choice) when they were struggling to sleep. This included describing the place in their minds, picturing the scenery and smells, as well as maintaining controlled regular breathing.

Sleep diary Continued from previous week

This module addressed common negative thoughts and feelings about insomnia (e.g., "I need 8 hours sleep or I will be too tired to function tomorrow").

Participants were asked to review their sleep diary data. If their sleep efficiency (i.e., a ratio of total spent asleep compared to the total amount of time in bed) was $80 \%$ or greater, they were instructed to loosen sleep restriction by going to bed 30 minutes earlier. However, if sleep efficiency was below $80 \%$, participants were instructed to go to bed 30 minutes later than last week.

Continued from previous week. sleep were put in place and practiced (e.g. "I will get out of bed and read for 10 minutes if I am struggling to fall asleep").

Sleep restriction reviewed in line with week four.

Continued from previous week.

Participants were instructed to get into bed and purposefully
A continuation of week four - new thought processes around As above. stay awake.

Sleep restriction Sleep restriction reviewed in line with week five. review

Sleep diary
As above.

As above.

Insomnia factor targeted

A range of behaviours, habits, and environments that can perpetuate insomnia, and inhibit sleep onset and maintenance.

The sleep diary was used to inform other intervention modules (e.g., sleep restriction) and helped participants to track progress.

Associations with the bed and bedroom as a place for associated with being unable to fall asleep, and/or stay asleep.

The increased physiological arousal associated with insomnia.

As above.

The consistent sleep/wake cycles that this method encourages helps to regulate the irregular sleep patterns commonly reported by those with insomnia.

Targets the increased cognitive arousal that is often associated with insomnia.

As above.

Addresses misconceptions that can feed into both night-time (e.g., becoming anxious the longer that sleep onset takes to occur) and day time arousal (e.g., attributing all day time fatigue to the previous night's sleep).

Behavioural goals and outcomes.

As above.

Reduces the 'performance anxiety' of falling asleep by asking participants to stay awake, thus reducing pre-sleep arousal, and inducing sleep onset.

As above.

As above. 
participants to the first of two relaxation methods - 'Progressive Muscle Relaxation', or PMR (i.e., contracting and relaxing muscle groups progressively over time in a set order). People experiencing insomnia often report high levels of cognitive and/or physiological arousal that can inhibit both getting to sleep and staying asleep (Harvey, 2002). PMR can be an effective method for reducing presleep arousal, particularly physiological arousal (McCallie et al., 2006; Sharma \& Andrade, 2012).

\section{Week three}

In week three, participants were asked to self-administer sleep restriction therapy, a form of controlled, systematic partial sleep deprivation that is intended to promote quicker sleep onset over the following days/weeks. Participants were asked to go to bed later than usual, at a time in line with how long they usually spend asleep rather than their desired total sleep time (i.e., someone who spends 9 hours in bed, but only 6 hours sleeping would be asked to restrict sleep to 6 hours in the first instance). The sleep restriction is titrated, so that if participants began to report falling asleep within an acceptable time frame (i.e., 15 to 20 minutes), the sleep restriction was reduced and the allotted time for sleep increased. Sleep restriction was used in conjunction with stimulus control, was restricted to a maximum of five hours sleep, and was reviewed at weekly intervals throughout the remainder of the intervention. The consistent sleep/ wake cycles that this method encourages are able regulate the irregular sleep patterns commonly reported by those with insomnia (Taylor \& Roane, 2010). Finally, in week three, participants were guided through an imaginative relaxation technique that they could use if they struggled to fall asleep, a method that has been associated with reduced cognitive arousal before bed (Sharma \& Andrade, 2012).

\section{Week four}

In week four, a number of misconceptions around sleep were discussed and participants were asked to reflect on whether and how these misconceptions might influence their own insomnia. Some common misconceptions about sleep that this module aimed to address were the idea that "I have to get at least eight hours sleep a night otherwise I will not function tomorrow", "I'm missing out on sleep, so I better have a nap to catch-up", and "I have lost control of my ability to fall asleep". This module explained how the thoughts feelings and behaviours that surround these misconceptions can impact on insomnia and create a vicious circle. For example, people who believe that they require at least eight hours to function the next day can 'clock-watch' and count the hours until their alarm goes off, which can subsequently lead to increased frustration and anxiety that starts the cycle again.

Participants were encouraged to break these cycles by completing an online CBT exercise. First, participants were asked to recall recent situations where they have struggled to sleep, before writing down their thoughts at the time (e.g., "if I do not fall asleep soon, then I will not function tomorrow"), how they felt about the situation ("I was frustrated and anxious because I had an important meeting the next day"), and how they responded ("I kept looking at my alarm and counting the hours until it went off, tossing and turning all the while"). Participants were then asked to think about potential more positive thoughts (e.g., "If I do not sleep eight hours, then I will be tired, but I will still function tomorrow"), feelings (e.g., "I am frustrated I cannot sleep straight away, but feeling anxious will only make it worse and I am confident that I will fall asleep soon"), and behaviours (e.g., "I will turn the clock away from me and try a relaxation exercise") to focus on instead. Participants were asked to complete these exercises whenever they experienced problems sleeping. Finally in week four, participants reviewed their sleep restriction. If sleep efficiency was $80 \%$ or greater, then they could loosen sleep restriction by going to bed 30 minutes earlier. However, if sleep efficiency was below $80 \%$, then participants were instructed to go to bed 30 minutes later than last week (providing that they allowed at least five hours' total sleep).

\section{Week five}

In week five, participants built on the techniques and exercises that they had learnt so far. No new content was introduced; however, participants were asked to complete the CBT exercises from week four with any new situations that they had encountered.

Week six

In the final week of the intervention, participants were encouraged to try paradoxical intention therapy if they were still struggling to sleep. Paradoxical intention essentially requires participants to get into bed and purposefully stay awake. In other words, this module prescribes the symptom as part of the solution; something that has been shown to improve sleep quality (Ascher \& Turner, 1979; DeBord, 1989; Perlis et al., 2010). The rationale behind paradoxical intention assumes that problems falling asleep are, in part, maintained by anxieties around the act of falling asleep itself. For example, cognitive models of insomnia suggest that the thoughts, feelings, and behaviours that arise from difficulties falling asleep can 'snowball' over time, with each previous negative experience contributing to future 'performance anxieties' around the act of falling asleep (Harvey, 2002). Paradoxical intention attempts to reduce this 'performance anxiety' around sleep onset by asking participants to do the opposite by staying awake (Perlis et al., 2010; Sharma \& Andrade, 2012).

\section{Intervention delivery}

The intervention was self-administered by the participants, with no contact from the research team, peers, or professionals (with the exception of the initial recruitment email). The website functioned on both laptops and personal computers, as well as tablets and mobile devices. Upon first login, participants were presented with an introductory homepage with instructions on how to use the website, and a navigation panel to the left of the screen showing an overview of each week of the intervention. 
Participants started with week one and subsequent weeks of the intervention were 'unlocked' following completion of the previous week's activities. Participants were able to return to any module they had previously 'unlocked', and could track their progress through the intervention using a progress bar that filled as each module was completed. Participants could also access the results of their completed sleep diaries (e.g., statistics on sleep efficiency, average bed and rise times etc.) in the form of tables and graphs by clicking on a "Sleep Stats" link at the top of each web page. The website was also able to send automated email reminders to participants who had not engaged with the website for one week, as well as those who had not completed sleep diary entries, or the follow-up questionnaires.

\section{Sleep-diary active control group}

Participants in both the intervention and the sleep diary group were asked to complete a sleep diary that asked them to record the time that they went to bed and got up each day, the number of times that they woke each night, how they felt in the morning (ranging from 1 - 'very tired' to 5 - 'very refreshed'), and their caffeine consumption.

\section{Wait-list control group}

The wait-list control group were given access to the full six-week intervention at the end of the trial; however, received no materials during the trial period.

\section{Required Sample Size}

G-Power v3.1 (Faul et al., 2007) was used to determine the number of participants that would be needed to detect a large-sized change in insomnia as a function of the intervention (based on a similar intervention, see Lancee et al., 2012). Power analysis, based on a significance level of $p<.05$ and $80 \%$ power, suggested that a minimum of 36 participants would be needed in each group (total $n=108$ across three groups) to detect an effect of this magnitude. Based on the assumption that we would be able to retain around one third of the sample 18-weeks post-intervention (a similar rate of attrition was reported by a similar study, see Lancee et al., 2012), the minimum sample size required at baseline was estimated at $n=319$ across the three groups.

\section{Randomisation}

Following completion of the baseline measures, participants were automatically randomised using an algorithm built into the study website with an approximate allocation ratio of $1: 1$. Immediately after randomisation, participants were given on screen instructions that detailed their allocated group (i.e., intervention, active control, or wait-list), along with instructions regarding what to do next.

\section{Blinding}

As randomisation to groups was conducted automatically by the study website, the research team was blinded to allocation. Furthermore, all outcome measures were collected online, and completed independently by each participant, meaning the research team were also blind to assessment. However, participants were not blind to allocation.

\section{Approach to Analysis}

The effect if the intervention on outcomes was examined using linear mixed effects modelling. This approach accounts for natural correlation between repeated measurements and allows all available data to be included, rather than, for example, excluding participants who only provided data at some time points (Gueorguieva \& Krystal, 2004)(Gueorguieva \& Krystal, 2004). The main effects of group, and time, as well as an interaction between group and time were as tested using Restricted Maximum Likelihood estimation (REML) and an unstructured covariance matrix. Given the relatively high level of missing data due to participant attrition (i.e., between $58 \%$ and $73 \%$ missing data across all time points), and that the data was not missing at random $\left(X^{2}(121)=204.16, p<0.001\right)$, multiple imputation is not advised (Jakobsen et al., 2017). Therefore, the analyses are based on actual available data and not imputed values.

\section{Software and Materials}

SPSS version 25 (IBM, 2017) was used to conduct the primary analysis and outcome measures were delivered online using Qualtrics.

\section{Ethics}

Ethical approval was granted by the Research Ethics Committee in the Department of Psychology at the University of Sheffield on the $15^{\text {th }}$ January 2014.

\section{Results \\ Participants}

Figure 1 shows the flow of participants through the trial. $N=457$ participants were screened for eligibility between April 2014 and February 2015, and $N=36$ participants were excluded: $N=12$ as their insomnia was better explained by a physical health problem; $N=6$ as they were currently using medication for a sleep complaint; and $N=18$ as they were currently receiving psychological therapy. $N=91$ participants did not complete any of the outcome measures at baseline, leaving $N=330$ participants who were randomised to one of the three groups. $N=94$ were randomised to receive the Sleep Healthy intervention, $N=100$ were randomised to the sleep diary only group, and $N=136$ were randomised to the wait-list control group.

\section{Participant Attrition}

There was a relatively high level of dropout throughout the study, with $N=224(68 \%)$ of the baseline sample dropping out of the study. Participants randomised to the intervention group were marginally more likely to drop-out of the study (85\%) than participants in the sleep diary group (76\%), OR $=1.80,95 \% \mathrm{CI}=0.87$ to $3.74, p=0.078$, and significantly more likely to drop out than participants in the wait-list control group (59\%), $\mathrm{OR}=4.00,95 \% \mathrm{CI}=2.06$ to 7.76, $p<0.001$. Similarly, participants allocated to the sleep diary group were 


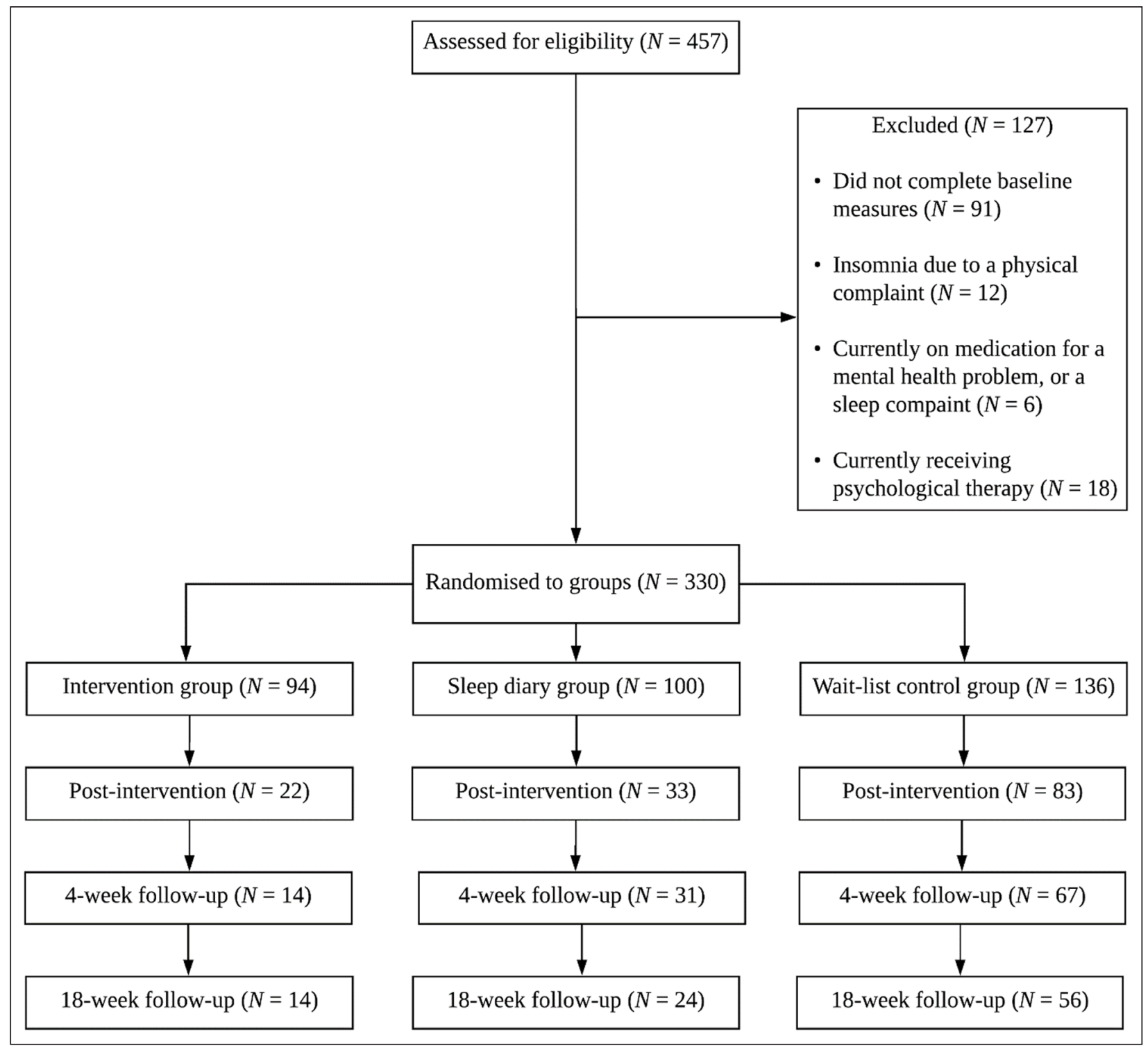

Figure 1: CONSORT Diagram Showing the Flow of Participants Through the Trial.

significantly more likely to drop-out than those allocated to the wait-list control group $(\mathrm{OR}=2.22,95 \% \mathrm{CI}=1.25$ to $3.93, p=0.007)$. Gender was not significantly associated with drop-out $(\mathrm{OR}=1.42,95 \% \mathrm{CI}=0.83$ to 2.41 , $p=0.123$ ); neither was severity of insomnia at baseline $(B=0.00, \mathrm{SE}=0.03, p=0.952)$, negative affect $(B=-0.02$, $\mathrm{SE}=0.01, p=0.086)$, or paranoia $(B=0.05, \mathrm{SE}=0.02$, $p=0.725)$. However, age did significantly predict drop out, with younger participants being more likely to dropout $(B=-1.14, \mathrm{SE}=0.13, p<0.001)$.

\section{Effect of the Intervention on Insomnia, Negative Affect, and Paranoia}

Figures 2, 3, and $\mathbf{4}$ display the estimated marginal mean scores over time (and by group) on insomnia, negative affect, and paranoia, and Table 2 shows the estimated marginal mean scores by group over time. There was a significant effect of time on insomnia $(F(3,123)=21.32$, $p<0.001, \mathrm{n}^{2}=0.34,95 \% \mathrm{Cl}=0.20$ to 0.44$)$, negative affect $\left(F(3,117)=3.90, p=0.011, \mathrm{\eta}^{2}=0.09,95 \% \mathrm{CI}=\right.$
0.01 to 0.18$)$, and paranoia $(F(3,100)=4.12, p=0.009$, $\eta^{2}=0.11,95 \% \mathrm{CI}=0.01$ to 0.21 , with all groups showing improvements in these outcomes at follow-up relative to baseline. However, there was no significant effect of group on levels of insomnia $(F(2,216)=1.11, p=0.330$, $\mathrm{\eta}^{2}=0.01,95 \% \mathrm{Cl}=0.00$ to 0.05$)$, negative affect $(F(2$, $255)=1.972, p=0.141, \mathrm{n}^{2}=0.02,95 \% \mathrm{Cl}=0.00$ to 0.05$)$, or paranoia $\left(F(2,259)=1.66, p=0.192, \eta^{2}=0.01,95 \%\right.$ $\mathrm{CI}=0.00$ to 0.05 ). Furthermore, there was no significant interaction between group and time on insomnia $(F(6$, 129) $=1.52, p=0.177, \eta^{2}=0.07,95 \% \mathrm{CI}=0.00$ to 0.12 ), negative affect $\left(F(6,135)=0.64, p=0.701, \mathrm{\eta}^{2}=0.03,95 \%\right.$ $\mathrm{CI}=0.00$ to 0.06$)$, or paranoia $(F(6,99)=1.29, p=0.268$, $\eta^{2}=0.07,95 \% \mathrm{CI}=0.00$ to 0.13 ). These findings suggest that insomnia, negative affect, and insomnia reduced over time irrespective group allocation. However, it is important to note that, although the analysis included all available data, due to participant attrition the analysis was statistically underpowered to detect an effect (see Table $\mathbf{2}$ for analysis $N$ at each time point). 


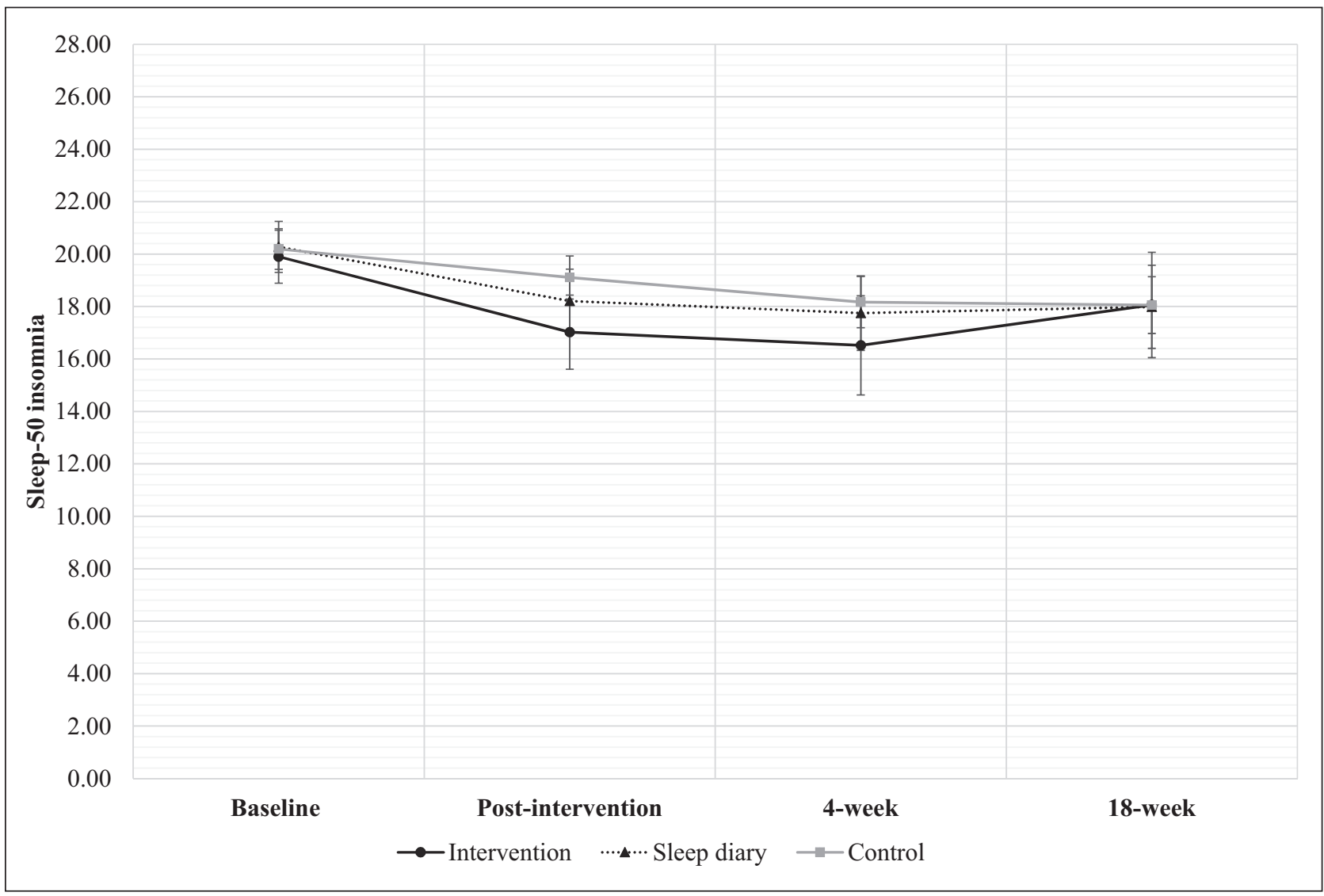

Figure 2: Estimated Marginal Mean Scores For Each Group Over Time for Insomnia.

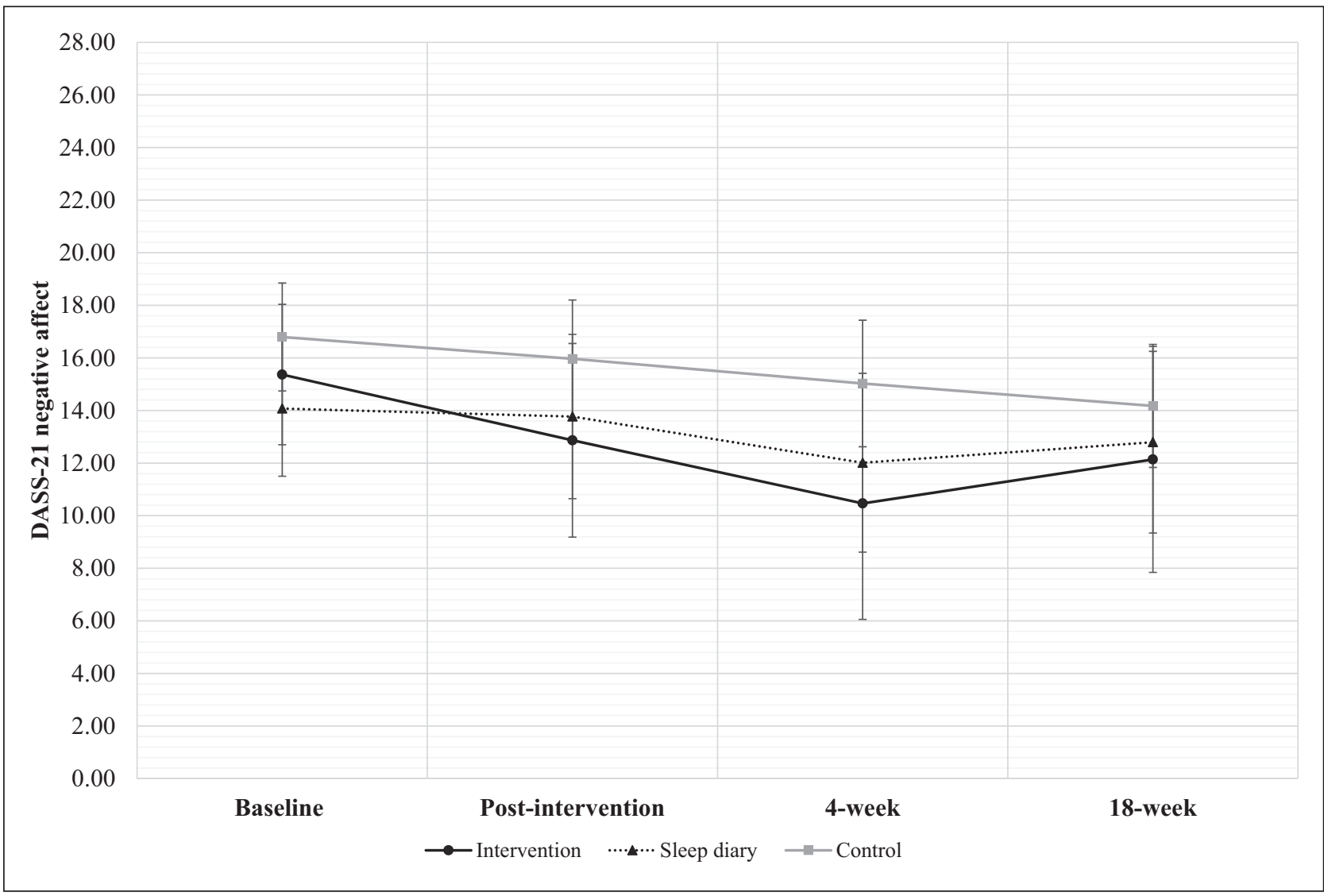

Figure 3: Estimated Marginal Mean Scores For Each Group Over Time for Negative Affect. 


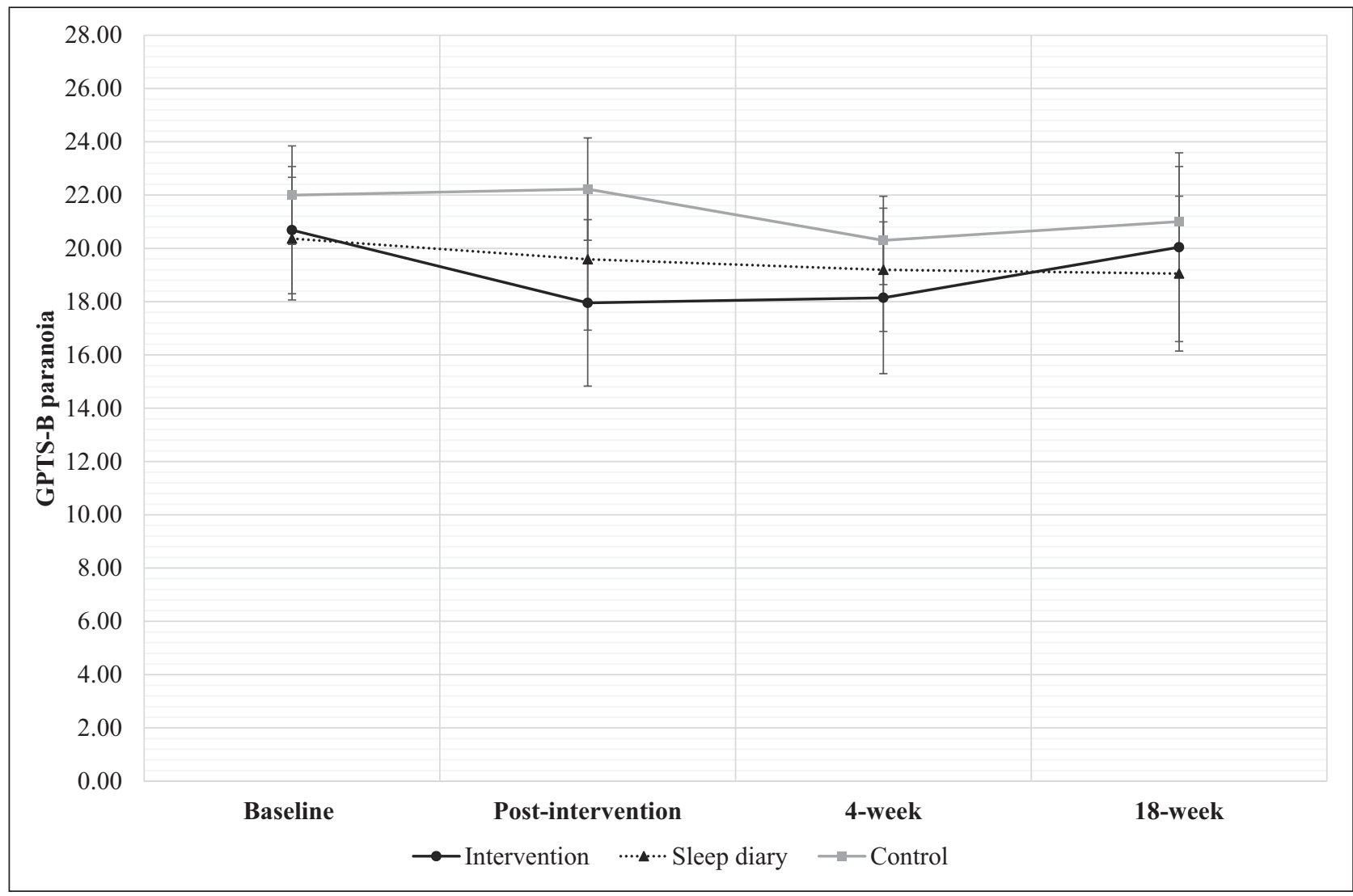

Figure 4: Estimated Marginal Mean Scores For Each Group Over Time for Paranoia.

Table 2: Estimated Marginal Means, Standard Errors and 95\% Confidence Intervals by Time and Group.

\begin{tabular}{|c|c|c|c|c|c|c|c|c|c|c|c|c|}
\hline & \multicolumn{4}{|c|}{ Intervention } & \multicolumn{4}{|c|}{ Sleep diary } & \multicolumn{4}{|c|}{ Wait-list } \\
\hline & $N$ & $M$ & $S E$ & $95 \% \mathrm{CI}$ & $N$ & $M$ & $S E$ & $95 \% \mathrm{CI}$ & $N$ & $M$ & $S E$ & $95 \% \mathrm{CI}$ \\
\hline Baseline & 94 & & & & 100 & & & & 136 & & & \\
\hline Insomnia & & 19.90 & 0.51 & 18.89 to 20.91 & & 20.28 & 0.49 & 19.30 to 21.25 & & 20.19 & 0.39 & 19.42 to 20.97 \\
\hline Negative affect & & 15.37 & 1.36 & 12.70 to 18.04 & & 14.07 & 1.31 & 11.50 to 16.65 & & 16.79 & 1.04 & 14.74 to 18.85 \\
\hline Paranoia & & 20.68 & 1.21 & 18.30 to 23.07 & & 20.37 & 1.17 & 18.06 to 22.67 & & 22.00 & 0.94 & 20.15 to 23.85 \\
\hline Post-intervention & 22 & & & & 33 & & & & 83 & & & \\
\hline Insomnia & & 17.02 & 0.72 & 15.61 to 18.44 & & 18.21 & 0.62 & 16.99 to 19.43 & & 19.10 & 0.42 & 18.28 to 19.93 \\
\hline Negative affect & & 12.87 & 1.87 & 9.18 to 16.55 & & 13.77 & 1.59 & 10.65 to 16.89 & & 15.97 & 1.13 & 13.73 to 18.20 \\
\hline Paranoia & & 17.95 & 1.59 & 14.83 to 21.08 & & 19.59 & 1.35 & 16.93 to 22.25 & & 22.22 & 0.97 & 20.30 to 24.14 \\
\hline 4-weeks & 14 & & & & 31 & & & & 67 & & & \\
\hline Insomnia & & 16.52 & 0.96 & 14.63 to 18.41 & & 17.75 & 0.72 & 16.33 to 19.17 & & 18.17 & 0.49 & 17.19 to 19.14 \\
\hline Negative affect & & 10.47 & 2.23 & 6.05 to 14.88 & & 12.01 & 1.72 & 8.61 to 15.41 & & 15.03 & 1.22 & 12.62 to 17.43 \\
\hline Paranoia & & 18.15 & 1.44 & 15.30 to 20.99 & & 19.19 & 1.17 & 16.88 to 21.51 & & 20.30 & 0.84 & 18.64 to 21.96 \\
\hline 18-weeks & 14 & & & & 24 & & & & 56 & & & \\
\hline Insomnia & & 18.06 & 1.02 & 16.05 to 20.07 & & 17.99 & 0.80 & 16.40 to 19.57 & & 18.06 & 0.55 & 16.97 to 19.14 \\
\hline Negative affect & & 12.14 & 2.17 & 7.84 to 16.44 & & 12.79 & 1.75 & 9.34 to 16.25 & & 14.17 & 1.18 & 11.83 to 16.51 \\
\hline Paranoia & & 20.14 & 1.79 & 16.50 to 23.59 & & 19.05 & 1.47 & 16.15 to 21.96 & & 21.00 & 1.05 & 18.93 to 23.07 \\
\hline
\end{tabular}

Note: Data presented are the estimated marginal mean scores (standard error). 


\section{Discussion}

The present research aimed to investigate whether an internet delivered self-help CBTi intervention designed to improve sleep could reduce insomnia, negative affect, and paranoid thinking. Our analysis suggested that the intervention did not improve insomnia, negative affect, or paranoid thinking, and that participants generally saw reductions in these outcomes over time irrespective of the group they were randomised too. However, the analyses were likely underpowered due to a relatively high level of dropout. Therefore, it cannot be ruled out that the null findings are due to a lack of statistical power, rather than the absence of an effect of the intervention on insomnia, negative affect, and/or paranoid thinking. Furthermore, the findings reported here are in contrast to recent, largescale randomised controlled trials that have examined the effect of improving sleep on paranoid thinking and affective experiences. For example, Freeman et al. (2017) randomised $N=3,755$ University students to receive an unguided digital CBTi intervention or usual care and found large-sized effects of the intervention on insomnia, small-sized effects on paranoid thinking, and small-tomedium sized effects on depression and anxiety (see also, Denis et al., 2019). Additionally, Christensen et al. (2016) reported that relative to an active control, participants receiving digital CBTi reported significantly lower depression symptoms at both post-intervention, and 6-months post-intervention. Consequently, although the present research is unable to add to a growing body of evidence that digital CBTi can lead to reductions in paranoia and negative affect, it does appear that improving insomnia as a route to improving mental health outcomes can be viable. However, it is also clear that dropout is a serious issue in online interventions in both the present study, and those reported elsewhere (Christensen et al., 2016; Eysenbach, 2005; Freeman et al., 2017; Lancee et al., 2012), and therefore represents a clear target for future research, which we expand on below.

\section{Understanding and tackling drop-out from online interventions}

Drop-out from online interventions is common and represents a major challenge to researchers, clinicians, and policy makers alike (Ahern \& Le Brocque, 2005; Geraghty et al., 2010). Indeed, Eysenbach (2005) states the case for a 'science of attrition' that distinguishes between nonusage attrition (i.e., not engaging with the intervention itself) and drop-out attrition (i.e., losing participants to follow-up). The present research was limited in that we did not measure the extent to which participants used the intervention; however, we found that younger age groups, and those randomised to the intervention and sleep diary groups were less likely to complete the follow-up measures than those allocated to the wait-list control group. Although data on the factors associated with attrition from online CBTi interventions is limited, evidence generally supports the notion that participants' age is associated with drop-out (Beatty \& Binnion, 2016; Davis \& Addis, 1999), with younger age groups often associated with increased attrition (Warden et al., 2009). Furthermore, as was found in the present research, participants allocated to intervention groups are generally more likely to dropout of health behaviour change trials than those allocated to control groups (Crutzen et al., 2015).

One explanation for the higher rate of drop out from the intervention, relative to control, conditions may be the increased demands that the intervention tasks placed on participants. For example, participants in the intervention conditions were asked to complete a sleep diary and submit this online on a daily basis. Such tasks may have been perceived as onerous and potentially abandoned if the participant encountered a barrier or felt that they were falling behind. One approach might be to augment interventions with Behaviour Change Techniques (BCTs) that are directly targeted at improving engagement (Garnett et al., 2015; Morrison et al., 2014; Perski et al., 2016; Webb et al., 2010). For example, participants might be prompted to form if-then plans (or 'implementation intentions', Gollwitzer, 1999) that link suitable opportunities to act (specified in the if part of the plan) with instrumental responses to those opportunities (specified in the then part of the plan). For example, a person using self-help CBTi may form a plan to complete their sleep diary as soon as they have finished breakfast: "As soon as I have finished my breakfast, then I will complete my sleep diary!" Implementation intentions have been shown to be an effective way of achieving intended goals (Gollwitzer \& Sheeran, 2006), and to aid engagement with self-help interventions (Shah, Hunt, Webb, \& Thompson, 2014; Varley et al., 2011).

The differential rate of attrition between the groups could also be due to raised expectations in the intervention group relative to controls, in that if the intervention does not meet expectations (i.e., by improving the target symptom/behaviour) participants dropout (or "implementation intentions', Gollwitzer, 1999). Participants allocated to control groups will likely not hold as high expectations (if any), therefore may be more likely to remain in the trial. The effect of expectations may have been exacerbated in the present research as some of the more effective components of CBTi were introduced later in the 6-week intervention, while components that have had less support were delivered early in week one. For example, Harvey, Inglis, and Espie (2002) reported that sleep hygiene education and relaxation exercises were not associated with treatment effect, while sleep scheduling techniques such as stimulus control (i.e., (re)associating the bedroom with sleep) and sleep restriction (limiting time spent in bed awake), and also cognitive restructuring of maladaptive thoughts about sleep were strong predictors of the effect of the intervention on sleep parameters. However, in the present intervention, these more effective components were not introduced until week two (stimulus control), week three (sleep restriction), and week four (cognitive restructuring). This problem may have been further compounded by the use of a 'tunnelled' navigation system, which meant that participants in the intervention group could only access next week's content after they had accessed the previous week's content in full. 


\section{Take Home Messages}

A relatively high rate of attrition meant that the present study did not have sufficient statistical power to provide a robust test of the impact of an online, self-help CBTi intervention on outcomes. Our primary take home message, therefore, is that attrition from digital CBTi interventions represents a major barrier to future trials aiming to test the effect in improving sleep on mental health outcomes and warrants consideration.

\section{Additional Files}

The additional files for this article can be found as follows:

- Supplementary File 1. Analysis package. DOI: https:// doi.org/10.5334/hpb.6.s1

- Supplementary File 2. Replication package. DOI: https://doi.org/10.5334/hpb.6.s2

\section{Competing Interests}

The authors have no competing interests to declare.

\section{References}

Ahern, K., \& Le Brocque, R. (2005). Methodological issues in the effects of attrition: Simple solutions for social scientists. Field Methods, 17(1), 53-69. DOI: https://doi.org/10.1177/1525822X04271006

Ascher, L. M., \& Turner, R. M. (1979). Paradoxical intention and insomnia: An experimental investigation. Behaviour Research and Therapy, 17(4), 408-411. DOI: https://doi.org/10.1016/0005-7967(79)90015-9

Aschim, B., Lundevall, S., Martinsen, E. W., \& Frich, J. C. (2011). General practitioners' experiences using cognitive behavioural therapy in general practice: A qualitative study. Scandinavian Journal of Primary Health Care, 29(3), 176-180. DOI: https:// doi.org/10.3109/02813432.2011.595582

Baglioni, C., Nanovska, S., Regen, W., Spiegelhalder, K., Feige, B., Nissen, C., Reynolds, C. F., III, \& Riemann, D. (2016). Sleep and mental disorders: A meta-analysis of polysomnographic research. Psychological Bulletin, 142(9), 969. DOI: https://doi. org/10.1037/bul0000053

Beatty, L., \& Binnion, C. (2016). A systematic review of predictors of, and reasons for, adherence to online psychological interventions. International Journal of Behavioral Medicine, 23(6), 776-794. DOI: https:// doi.org/10.1007/s12529-016-9556-9

Benca, R. M., Obermeyer, W. H., Thisted, R. A., \& Gillin, J. C. (1992). Sleep and psychiatric disorders: A meta-analysis. Archives of General Psychiatry, 49(8), 651-668. DOI: https://doi.org/10.1001/ archpsyc.1992.01820080059010

Bootzin, R. R. (1972). Stimulus control treatment for insomnia. Proceedings of the American Psychological Association, 7, 395-396.

Cavanagh, K. (2014). Geographic Inequity in the Availability of Cognitive Behavioural Therapy in England and Wales: A 10-Year Update. Behavioural and Cognitive Psychotherapy, 42(4), 497-501. DOI: https://doi.org/10.1017/S1352465813000568
Cheng, S. K., \& Dizon, J. (2012). Computerised cognitive behavioural therapy for insomnia: A systematic review and meta-analysis. Psychotherapy and Psychosomatics, 81(4), 206-216. DOI: https://doi. org/10.1159/000335379

Christensen, H., Batterham, P. J., Gosling, J. A., Ritterband, L. M., Griffiths, K. M., Thorndike, F. P., Glozier, N., O'Dea, B., Hickie, I. B., \& Mackinnon, A. J. (2016). Effectiveness of an online insomnia program (SHUTi) for prevention of depressive episodes (the GoodNight Study): A randomised controlled trial. The Lancet Psychiatry, 3(4), 333-341. DOI: https://doi.org/10.1016/ S2215-0366(15)00536-2

Crutzen, R., Viechtbauer, W., Spigt, M., \& Kotz, D. (2015). Differential attrition in health behaviour change trials: A systematic review and meta-analysis. Psychology \& Health, 30(1), 122-134. DOI: https:// doi.org/10.1080/08870446.2014.953526

Cuijpers, P., Donker, T., van Straten, A., Li, J., \& Andersson, G. (2010). Is guided self-help as effective as face-to-face psychotherapy for depression and anxiety disorders? A systematic review and meta-analysis of comparative outcome studies. Psychological Medicine, 40(12), 1943-1957. DOI: https://doi.org/10.1017/S0033291710000772

Davis, M. J., \& Addis, M. E. (1999). Predictors of attrition from behavioral medicine treatments. Annals of Behavioral Medicine, 21(4), 339-349. DOI: https:// doi.org/10.1007/BF02895967

DeBord, J. B. (1989). Paradoxical interventions: A review of the recent literature. Journal of Counseling \& Development, 67(7), 394-398. DOI: https://doi. org/10.1002/j.1556-6676.1989.tb02099.x

Denis, D., Eley, T. C., Rijsdijk, F., Zavos, H., Keers, R., Espie, C. A., Luik, A. I., Badini, I., Derveeuw, S., \& Hodsoll, J. (2019). Is digital cognitive behavioural therapy for insomnia effective in treating subthreshold insomnia: A pilot RCT. Sleep Medicine, 66, 174-183. DOI: https://doi.org/10.1016/j.sleep. 2019.10.007

Eysenbach, G. (2005). The Law of Attrition. Journal of Medical Internet Research, 7(1). DOI: https://doi. org/10.2196/jmir.7.1.e11

Faul, F., Erdfelder, E., Lang, A.-G., \& Buchner, A. (2007). $G^{*}$ Power 3: A flexible statistical power analysis program for the social, behavioral, and biomedical sciences. Behavior Research Methods, 39(2), 175191. DOI: https://doi.org/10.3758/BF03193146

Fowler, D., Hodgekins, J., Garety, P., Freeman, D., Kuipers, E., Dunn, G., Smith, B., \& Bebbington, P. E. (2012). Negative Cognition, Depressed Mood, and Paranoia: A Longitudinal Pathway Analysis Using Structural Equation Modeling. Schizophrenia Bulletin, 38(5), 1063-1073. DOI: https://doi.org/ 10.1093/schbul/sbr019

Freeman, D., Brugha, T., Meltzer, H., Jenkins, R., Stahl, D., \& Bebbington, P. (2010). Persecutory ideation and insomnia Findings from the second British National Survey Of Psychiatric Morbidity. Journal 
of Psychiatric Research, 44(15), 1021-1026. DOI: https://doi.org/10.1016/j.jpsychires.2010.03.018

Freeman, D., Garety, P. A., Bebbington, P. E., Smith, B., Rollinson, R., Fowler, D., Kuipers, E., Ray, K., \& Dunn, G. (2005). Psychological investigation of the structure of paranoia in a non-clinical population. British Journal of Psychiatry, 186, 427-435. DOI: https://doi.org/10.1192/bjp.186.5.427

Freeman, D., Pugh, K., Vorontsova, N., \& Southgate, L. (2009). Insomnia and paranoia. Schizophrenia Research, 108(1-3), 280-284. DOI: https://doi. org/10.1016/j.schres.2008.12.001

Freeman, D., Sheaves, B., Goodwin, G. M., Yu, L.-M., Nickless, A., Harrison, P. J., Emsley, R., Luik, A. I., Foster, R. G., \& Wadekar, V. (2017). The effects of improving sleep on mental health (OASIS): A randomised controlled trial with mediation analysis. The Lancet Psychiatry, 4(10), 749-758. DOI: https:// doi.org/10.1016/S2215-0366(17)30328-0

Freeman, D., Stahl, D., McManus, S., Meltzer, H., Brugha, T., Wiles, N., \& Bebbington, P. (2012). Insomnia, worry, anxiety and depression as predictors of the occurrence and persistence of paranoid thinking. Social Psychiatry and Psychiatric Epidemiology, 47(8), 1195-1203. DOI: https://doi. org/10.1007/s00127-011-0433-1

Freeman, D., Waite, F., Startup, H., Myers, E., Lister, R., McInerney, J., Harvey, A. G., Geddes, J., Zaiwalla, Z., \& Luengo-Fernandez, R. (2015). Efficacy of cognitive behavioural therapy for sleep improvement in patients with persistent delusions and hallucinations (BEST): A prospective, assessorblind, randomised controlled pilot trial. The Lancet Psychiatry, 2(11), 975-983. DOI: https://doi.org/ 10.1016/S2215-0366(15)00314-4

Garnett, C., Crane, D., West, R., Brown, J., \& Michie, S. (2015). Identification of behavior change techniques and engagement strategies to design a smartphone app to reduce alcohol consumption using a formal consensus method. JMIR MHealth and UHealth, 3(2). DOI: https://doi.org/10.2196/ mhealth.3895

Gellatly, J., Bower, P., Hennessy, S., Richards, D., Gilbody, S., \& Lovell, K. (2007). What makes self-help interventions effective in the management of depressive symptoms? Meta-analysis and meta-regression. Psychological Medicine, 37(9), 1217-1228. DOI: https://doi.org/10.1017/S003329 1707000062

Geraghty, A. W. A., Wood, A. M., \& Hyland, M. E. (2010). Attrition from self-directed interventions: Investigating the relationship between psychological predictors, intervention content and dropout from a body dissatisfaction intervention. Social Science \& Medicine, 71(1), 30-37. DOI: https://doi. org/10.1016/j.socscimed.2010.03.007

Gollwitzer, P. M. (1999). Implementation intentions: Strong effects of simple plans. American Psychologist, 54(7), 493. DOI: https://doi.org/10.1037/ 0003-066X.54.7.493
Green, C. E. L., Freeman, D., Kuipers, E., Bebbington, P., Fowler, D., Dunn, G., \& Garety, P. A. (2008). Measuring ideas of persecution and social reference: The Green et al. Paranoid Thought Scales (GPTS). Psychological Medicine, 38(1), 101-111. DOI: https://doi.org/10.1017/S0033291707001638

Grezellschak, S., Jansen, A., \& Westermann, S. (2017). Emotion regulation in patients with psychosis: A link between insomnia and paranoid ideation? Journal of Behavior Therapy and Experimental Psychiatry, 56, 27-32. DOI: https://doi.org/10.1016/j.jbtep. 2016.08.001

Gueorguieva, R., \& Krystal, J. H. (2004). Move over anova: Progress in analyzing repeated-measures data and its reflection in papers published in the archives of general psychiatry. Archives of General Psychiatry, 61(3), 310-317. DOI: https://doi.org/10.1001/ archpsyc.61.3.310

Harvey, A. G. (2002). A cognitive model of insomnia. Behaviour Research and Therapy, 40(8), 869-893. DOI: https://doi.org/10.1016/S0005-7967(01)00061-4

Harvey, L., Inglis, S. J., \& Espie, C. A. (2002). Insomniacs' reported use of CBT components and relationship to long-term clinical outcome. Behaviour Research and Therapy, 40(1), 75-83. DOI: https://doi.org/ 10.1016/S0005-7967(01)00004-3

Henry, J. D., \& Crawford, J. R. (2005). The short-form version of the Depression Anxiety Stress Scales (DASS-21): Construct validity and normative data in a large non-clinical sample. British Journal of Clinical Psychology, 44(2), 227-239. DOI: https:// doi.org/10.1348/014466505X29657

Ho, F. Y.-Y., Chung, K.-F., Yeung, W.-F., Ng, T. H., Kwan, K.-S., Yung, K.-P., \& Cheng, S. K. (2015). Self-help cognitive-behavioral therapy for insomnia: A metaanalysis of randomized controlled trials. Sleep Medicine Reviews, 19, 17-28. DOI: https://doi. org/10.1016/j.smrv.2014.06.010

IBM. (2017). IBM SPSS Statistics for Windows. IBM Corp.

Jakobsen, J. C., Gluud, C., Wetterslev, J., \& Winkel, P. (2017). When and how should multiple imputation be used for handling missing data in randomised clinical trials-a practical guide with flowcharts. BMC Medical Research Methodology, 17(1), 162. DOI: https://doi.org/10.1186/s12874-017-0442-1

Lancee, J., van den Bout, J., van Straten, A., \& Spoormaker, V. I. (2012). Internet-delivered or mailed self-help treatment for insomnia?: A randomized waiting-list controlled trial. Behaviour Research and Therapy, 50(1), 22-29. DOI: https:// doi.org/10.1016/j.brat.2011.09.012

Lincoln, T. M., Lange, J., Burau, J., Exner, C., \& Moritz, S. (2010). The Effect of State Anxiety on Paranoid Ideation and Jumping to Conclusions. An Experimental Investigation. Schizophrenia Bulletin, 36(6), 1140-1148. DOI: https://doi.org/10.1093/schbul/ sbp029

Lincoln, T. M., Peter, N., Schaefer, M., \& Moritz, S. (2009). Impact of stress on paranoia: An experimental investigation of moderators and mediators. 
Psychological Medicine, 39(7), 1129-1139. DOI: https://doi.org/10.1017/S0033291708004613

McCallie, M. S., Blum, C. M., \& Hood, C. J. (2006). Progressive muscle relaxation. Journal of Human Behavior in the Social Environment, 13(3), 51-66. DOI: https://doi.org/10.1300/J137v13n03_04

Morin, C. M. (1993). Insomnia: Psychological assessment and management. Guilford press.

Morrison, L., Moss-Morris, R., Michie, S., \& Yardley, L. (2014). Optimizing engagement with Internet-based health behaviour change interventions: Comparison of self-assessment with and without tailored feedback using a mixed methods approach. British Journal of Health Psychology, 19(4), 839-855. DOI: https://doi.org/10.1111/bjhp.12083

Myers, E., Startup, H., \& Freeman, D. (2011). Cognitive behavioural treatment of insomnia in individuals with persistent persecutory delusions: A pilot trial. Journal of Behavior Therapy and Experimental Psychiatry, 42(3), 330-336. DOI: https://doi. org/10.1016/j.jbtep.2011.02.004

Ohayon, M. M. (2002). Epidemiology of insomnia: What we know and what we still need to learn. Sleep Medicine Reviews, 6(2), 97-111. DOI: https://doi. org/10.1053/smrv.2002.0186

Okajima, I., Komada, Y., \& Inoue, Y. (2011). A metaanalysis on the treatment effectiveness of cognitive behavioral therapy for primary insomnia. Sleep and Biological Rhythms, 9(1), 24-34. DOI: https://doi. org/10.1111/j.1479-8425.2010.00481.x

Perlis, M. L., Aloia, M., \& Kuhn, B. (2010). Behavioral treatments for sleep disorders: A comprehensive primer of behavioral sleep medicine interventions. Academic Press.

Perlis, M. L., \& Youngstedt, S. D. (2000). The diagnosis of primary insomnia and treatment alternatives. Comprehensive Therapy, 26(4), 298-306. DOI: https://doi.org/10.1007/s12019-000-0033-6

Perski, O., Blandford, A., West, R., \& Michie, S. (2016). Conceptualising engagement with digital behaviour change interventions: A systematic review using principles from critical interpretive synthesis. Translational Behavioral Medicine, 7(2), 254-267. DOI: https://doi.org/10.1007/s13142-016-0453-1

Rehman, A., Gumley, A., \& Biello, S. (2018). Sleep quality and paranoia: The role of alexithymia, negative emotions and perceptual anomalies. Psychiatry Research, 259, 216-222. DOI: https://doi.org/10. 1016/j.psychres.2017.09.066

Riemann, D., \& Perlis, M. L. (2009). The treatments of chronic insomnia: A review of benzodiazepine receptor agonists and psychological and behavioral therapies. Sleep Medicine Reviews, 13(3), 205-214. DOI: https://doi.org/10.1016/j. smrv.2008.06.001

Scott, A. J., Rowse, G., \& Webb, T. L. (2017). A structural equation model of the relationship between insomnia, negative affect, and paranoid thinking. PLOS ONE, 12(10), e0186233. DOI: https://doi. org/10.1371/journal.pone.0186233
Scott, A. J., Webb, T. L., \& Rowse, G. (2015). Self-help interventions for psychosis: A meta-analysis. Clin Psychol Rev, 39, 96-112. DOI: https://doi. org/10.1016/j.cpr.2015.05.002

Shapiro, D. A., Cavanagh, K., \& Lomas, H. (2003). Geographic inequity in the availability of cognitive behavioural therapy in England and Wales. Behavioural and Cognitive Psychotherapy, 31(2), 185-192. DOI: https://doi.org/10.1017/ S1352465803002066

Sharma, M. P., \& Andrade, C. (2012). Behavioral interventions for insomnia: Theory and practice. Indian Journal of Psychiatry, 54(4), 359. DOI: https://doi.org/10.4103/0019-5545.104825

Smith, M. T., Perlis, M. L., Park, A., Smith, M. S., Pennington, J., Giles, D. E., \& Buysse, D. J. (2002). Comparative meta-analysis of pharmacotherapy and behavior therapy for persistent insomnia. American Journal of Psychiatry, 159(1), 5-11. DOI: https://doi. org/10.1176/appi.ajp.159.1.5

Spoormaker, V. I., Verbeek, I., van den Bout, J., \& Klip, E. C. (2005). Initial validation of the SLEEP50 questionnaire. Behavioral Sleep Medicine, 3(4), 227-246. DOI: https://doi.org/10.1207/ s15402010bsm0304_4

Taylor, D. J., \& Roane, B. M. (2010). Treatment of insomnia in adults and children: A practice-friendly review of research. Journal of Clinical Psychology, 66(11), 1137-1147. DOI: https://doi.org/10.1002/ jclp.20733

Trauer, J. M., Qian, M. Y., Doyle, J. S., Rajaratnam, S. M., \& Cunnington, D. (2015). Cognitive behavioral therapy for chronic insomnia: A systematic review and meta-analysis. Annals of Internal Medicine, 163(3), 191-204. DOI: https://doi.org/10.7326/ M14-2841

Unbehaun, T., Spiegelhalder, K., Hirscher, V., \& Riemann, D. (2010). Management of insomnia: Update and new Approaches. Nature and Science of Sleep, 2, 127-138. DOI: https://doi.org/10.2147/ NSS.S6642

van Straten, A., \& Cuijpers, P. (2009). Self-help therapy for insomnia: A meta-analysis. Sleep Medicine Reviews, 13(1), 61-71. DOI: https://doi. org/10.1016/j.smrv.2008.04.006

van Straten, A., Cuijpers, P., Smit, F., Spermon, M., \& Verbeek, I. (2009). Self-help treatment for insomnia through television and book: A randomized trial. Patient Education and Counseling, 74(1), 29-34. DOI: https://doi.org/10.1016/j.pec.2008.07.050

Warden, D., Rush, A. J., Carmody, T. J., Kashner, T. M., Biggs, M. M., Crismon, M. L., \& Trivedi, M. H. (2009). Predictors of attrition during one year of depression treatment: A roadmap to personalized intervention. Journal of Psychiatric Practice, 15(2), 113. DOI: https://doi.org/10.1097/01.pra. 0000348364.88676 .83

Webb, T. L., Joseph, J., Yardley, L., \& Michie, S. (2010). Using the internet to promote health behavior change: A systematic review and meta-analysis 
of the impact of theoretical basis, use of behavior change techniques, and mode of delivery on efficacy. Journal of Medical Internet Research, 12(1). DOI: https://doi.org/10.2196/jmir.1376

Wiebe, E., \& Greiver, M. (2005). Using cognitive behavioural therapy in practice: Qualitative study of family physicians' experiences. Canadian Family Physician Medecin de Famille Canadien, 51, 992-993.

Zachariae, R., Lyby, M. S., Ritterband, L. M., \& O'Toole, M. S. (2016). Efficacy of internet-delivered cognitive-behavioral therapy for insomnia-a systematic review and meta-analysis of randomized controlled trials. Sleep Medicine Reviews, 30, 1-10. DOI: https://doi.org/10.1016/j.smrv.2015.10.004

\section{Peer Review Comments}

Health Psychology Bulletin has blind peer review, which is unblinded upon article acceptance. The editorial history of this article can be downloaded here:

- PR File 1. Peer Review History. DOI: https://doi.org/ 10.5334/hpb.6.pr1

How to cite this article: Scott, A. J., Rowse, G., \& Webb, T. L. (2020). The Effect of an Online Self-Help Cognitive Behavioural Intervention for Insomnia on Negative Affect and Paranoia: A Randomised Controlled Trial. Health Psychology Bulletin, 4(1), 39-52. DOI: https://doi.org/10.5334/hpb.6

Submitted: 05 May 2017 Accepted: 30 September $2020 \quad$ Published: 06 October 2020

Copyright: $\odot 2020$ The Author(s). This is an open-access article distributed under the terms of the Creative Commons Attribution 4.0 International License (CC-BY 4.0), which permits unrestricted use, distribution, and reproduction in any medium, provided the original author and source are credited. See http://creativecommons.org/licenses/by/4.0/.

] $\mathbf{u}[\quad$ Health Psychology Bulletin is a peer-reviewed open access journal published by Ubiquity OPEN ACCESS $\boldsymbol{\gamma}$ 